\title{
Filigrane
}

Écoutes psychanalytiques

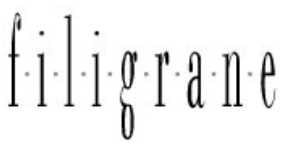

\section{Genres, subjectivités et corps au-delà de la binarité}

\section{Denise Medico}

Volume 28, numéro 1, 2019

Identités $^{2}$. Qui suis-je?

URI : https://id.erudit.org/iderudit/1064597ar

DOI : https://doi.org/10.7202/1064597ar

Aller au sommaire du numéro

Éditeur(s)

Santé mentale et société

ISSN

1192-1412 (imprimé)

1911-4656 (numérique)

Découvrir la revue

\section{Citer cet article}

Medico, D. (2019). Genres, subjectivités et corps au-delà de la binarité. Filigrane, 28(1), 57-71. https://doi.org/10.7202/1064597ar

\section{Résumé de l'article}

Les personnes trans interpellent et les débats théoriques et politiques les concernant s'enferment trop souvent dans un chiasme entre une apologie de l'identité et de la libération et une mise en garde sur la perte des repères immémoriaux. Mais comment en sortir si ce n'est en tentant de comprendre l'expérience vécue des personnes dont on parle ? Ce texte propose d'intégrer données empiriques, expériences cliniques, phénoménologie et théories contemporaines sur le genre pour comprendre l'expérience de soi des personnes trans et non binaires. De par leur existence, les personnes trans mettent en lumière la présence de ce qui est en fait déjà là : la subversion du système de genre binaire, mais aussi la question des liens entre modifications corporelles et construction du Sujet. Les subjectivités trans ne sont pas que la réplique d'une idée du féminin et du masculin, elles expriment autre chose, de l'ordre de la multiplicité, du devenir, des relations, de l'importance du ressenti corporel. Les corps y sont envisagés comme des matérialités signifiantes, origines et lieux du devenir trans. Découlant de la phénoménologie, le nouveau matérialisme est proposé comme outil conceptuel permettant d'appréhender ces matérialités affectées, signifiantes et transformatives.
Ce document est protégé par la loi sur le droit d'auteur. L'utilisation des services d’Érudit (y compris la reproduction) est assujettie à sa politique d'utilisation que vous pouvez consulter en ligne.

https://apropos.erudit.org/fr/usagers/politique-dutilisation/ 


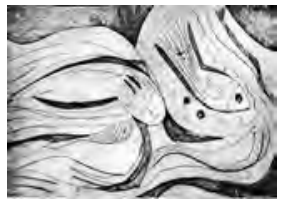

\title{
Genres, subjectivités et corps au-delà de la binarité
}

\section{Denise Medico}

Résumé: Les personnes trans interpellent et les débats théoriques et politiques les concernant s'enferment trop souvent dans un chiasme entre une apologie de l'identité et de la libération et une mise en garde sur la perte des repères immémoriaux. Mais comment en sortir si ce n'est en tentant de comprendre l'expérience vécue des personnes dont on parle? Ce texte propose d'intégrer données empiriques, expériences cliniques, phénoménologie et théories contemporaines sur le genre pour comprendre l'expérience de soi des personnes trans et non binaires. De par leur existence, les personnes trans mettent en lumière la présence de ce qui est en fait déjà là: la subversion du système de genre binaire, mais aussi la question des liens entre modifications corporelles et construction du Sujet. Les subjectivités trans ne sont pas que la réplique d'une idée du féminin et du masculin, elles expriment autre chose, de l'ordre de la multiplicité, du devenir, des relations, de l'importance du ressenti corporel. Les corps y sont envisagés comme des matérialités signifiantes, origines et lieux du devenir trans. Découlant de la phénoménologie, le nouveau matérialisme est proposé comme outil conceptuel permettant d'appréhender ces matérialités affectées, signifiantes et transformatives.

Mots clés : trans; genre; non binaire; subjectivité; corps; nouveau matérialisme.

\begin{abstract}
Trans people challenge society, and theoretical and political debates are too often confined within a chiasmus that articulates an apology of identity and liberation and a warning about the loss of immemorial references. But how can we get out of this opposition if not by trying to understand the lived experience of the people we are talking about? This text seeks to integrate empirical data, clinical experiences, phenomenology and contemporary gender theories to understand the experience of the self of trans and non-binary people. In their existence, trans people highlight what is already present: the subversion of the binary gender system, but also the connections between body modifications and the construction of the Subject. Trans subjectivities are not a mere replica of an idea about the feminine and the masculine, they express something beyond and speak of multiplicity, of becoming, of relationships and of the importance of felt body. The bodies are considered as signifying materialities and as the origin and place of trans becoming. Arising from phenomenology, new materialism proposes a conceptual tool to apprehend these affected, meaningful and transformative materialities.
\end{abstract}

Key words: trans; gender; non-binary; subjectivity; body; new materialism. 
L'idée de Foucault est non seulement que nous sommes certes toujours en relation à ces normes, mais que toute relation au régime de vérité sera toujours en même temps une relation à moi-même. Une opération de critique ne peut avoir lieu sans cette dimension réflexive. Remettre en question un régime de vérité, lorsque ce régime de vérité gouverne la subjectivation, c'est remettre en question la vérité de moi-même et, en fait, ma propre capacité à dire la vérité sur moi, à rendre compte de moi. (Butler, 2007, p. 22-23)

\section{«Malades, pervers, contaminés ! "}

es jeunes sont de plus en plus nombreux à s'identifier comme des per- sonnes trans ${ }^{1}$, à ne s'identifier ni comme homme, ni comme femme, mais comme des personnes non binaires et parfois neutres dans le genre (Richards et al., 2016). Même la revue Science (2018) en parle, car le débat s'envenime dans le petit monde de la «science trans», illustrant combien les travaux de recherche, comme les positions des cliniciens, sont empreints de politique, ce qui est d'autant plus visible lorsqu'ils abordent des objets, ou des sujets, qui sont en soi politiques.

La suspicion plane: s'agirait-il d'une contagion sociale? un nouveau virus qui se propagerait par réseaux sociaux? Cette hypothèse baptisée «ROGD» (rapid onset gender dysphoria) se base sur le fait que ces demandes semblent soudaines. Elle propose l'idée d'une contagion sociale que Michael Bailey qualifie même d' «épidémie».

Ce n'est pas la première fois - et avec les mêmes personnes - que ce type de discours s'exerce. Je rappelle la fameuse controverse autour du livre du même auteur paru en 2003. Elle portait sur une autre hypothèse associant genre, orientation sexuelle et sexualité, se basant sur le concept d'autogynéphilie proposé par Ray Blanchard (1989) et qui réfère à un amour ou une excitation face à l'image de soi en femme. L'autogynéphilie serait une forme de paraphilie que l'on retrouve encore dans la $5^{\mathrm{e}}$ édition du DSM dans le trouble paraphilique fétichiste sous forme de sous-catégorie. Pour résumer les positions précédemment tenues par Bailey, les désirs sexuels des personnes assignées au masculin seraient une origine ou une motivation à leurs demandes de transition de genre («transsexuelle» selon les mots de l'auteur). Comme il le dit lui-même, sans métaphore probablement, il cherche «le chaînon manquant» entre l'homme hétérosexuel et la 
personne transsexuelle: il le trouve dans l'homosexuel efféminé, reconstruisant ainsi une sorte de théorie de l'évolution pour l'homme dans sa masculinité sur un modèle de darwinisme social ${ }^{2}$ où le sommet de l'évolution serait probablement l'homme hétérosexuel masculin. L'idée qui sous-tend donc l'hypothèse de l'autogynéphilie est que la sexualité serait la motivation des demandes de transition transsexuelle, c'est-à-dire, pour être bref, une demande perverse qui viserait à se créer comme son propre objet d'excitation et ainsi se satisfaire de soi en circuit fermé. Un point de vue des plus pathologiques qui devrait soulever la question du narcissisme, mais qui ne le fait pas.

Si l'intérêt pour la sexualité a traversé l'histoire du concept médical de «transsexualisme» c'est qu'elle a surtout été utilisée par les psys comme un critère pour établir des diagnostics différentiels et effectuer le gatekeeping des traitements médicaux de confirmation de genre (Hérault, 2010; Medico, à paraître). Dans les faits, elle a surtout servi à ostraciser les personnes trans qui ne se conformaient pas à une idée de ce qu'était la transsexualité, à savoir l'idée médicale et clinique qui a déjà été passablement déconstruite et explicitée sous l'appellation de transsexual narrative (Hirschauer, 1998). Dans les domaines cliniques qui ont encore du mal à entériner la question d'une non pathologie et donc des droits des citoyens trans à disposer de leurs vies, de leurs identités et de leurs corps, les positions restent figées sur la question diagnostique et la recherche d'étiologies. Mais si cette recherche étiologique s'avère vaine, ce sont surtout les discours et les enjeux politiques liés au pouvoir de qui a le droit de choisir (les modifications corporelles médicalement induite) qui frappent dans les positions d'hier comme d'aujourd'hui. Dans les positions cliniques et médicales, un aspect reste toutefois stable: on n'entend pas et ne valide pas la parole des personnes concernées, on entend idée/ identité/changement de sexe quand les personnes disent ressenti/corps/non binarité.

La question qui m'interpelle est «pourquoi n'entend-on pas?» et aussi celle de savoir si la réponse ne serait pas plus politique que réellement un problème d'oreille ou d'interprétation? Pour comprendre le point de vue de Bailey que j'utilise ici comme victime émissaire ou récit mythologique, il pourrait être pertinent de resituer la mission sociale que se donne l'auteur, soit prévenir l'Amérique de l'augmentation du nombre de personnes trans. Je tiens d'ailleurs à souligner que, étonnamment, même ceux qui critiquent l'engagement militant d'autres chercheurs ou cliniciens se sentent des missions sociales qui dépassent largement leur pratique et leurs champs d'action 
de cliniciens ou de chercheurs. Donc, si l'on veut résumer rapidement l'évolution des hypothèses médicales et scientifiques de ceux qui crient au danger: les personnes trans ont passé du statut de malades (transsexualisme, dysphorie de genre) ou de pervers sexuels (paraphilie autogynéphilique) à celui de contaminés (ROGD). Ou alors on peut aussi se dire qu'elles sortent maintenant du déni d'existence et de l'invisibilité sociale (Namaste, 2000) pour affirmer leurs droits et différences et qu’il faut penser avec cette réalité qui est un changement de monde et pas seulement de mode.

Mais il faut peut-être penser plus largement ce changement et pas seulement avec l'hypothèse de la libération des identités ou à l'inverse d'un complot de militants trans. Les personnes trans, neutres et non binaires révèlent des modifications plus profondes des subjectivités et de l'être-ensemble contemporains. Iels ${ }^{3}$ existent. Pour nous les psys, qu'est-ce que cela signifierait, si nous cessions de vouloir penser comme si rien ne changeait, avec des outils conceptuels qui sont de toute évidence non appropriés puisque les personnes qu'ils sont censés aider et penser ne s'y reconnaissent pas, voire s'en sentent maltraitées (Sironi, 2003)? Pour cela, il nous faut écouter les vies qui se racontent. Cela signifie très concrètement qu'il ne faut pas oublier la réalité des enjeux de pouvoir qui sont indissociables de la notion de genre, des expériences de vie, de la possibilité de faire de la recherche ou de la clinique, des positions que nous avons chacun de nous dans l'espace social et qui nous attribuent des droits et des entraves.

\section{Points de vue, méthodes et concepts pour une clinique critique phénoménologique}

Ma question est donc «comment construire une théorie qui fonctionne, qui parle de et avec ceux dont elle parle?», qui ne se replie pas sur la méthode de l'exclusion pour cacher les différences, sur la pathologie, l'accusation de perversion ou la maladie infectieuse. Je propose dans ce texte un condensé de ce que j'ai compris quant à l'expérience des personnes trans en 18 ans de fréquentation, à travers deux terrains de recherche déjà publiés, un autre sur les jeunes trans et leurs parents en cours d'analyse, une revue critique de la littérature sur la sexualité des personnes trans, ainsi que mon expérience clinique dans l'accompagnement de personnes trans pendant plus de 15 ans, la supervision d'un réseau de thérapeutes et médecins et l'engagement dans la défense des droits des personnes trans (Medico, 2014, 2016; Medico et Volkmar, 2016; Medico et al., 2009). Ma méthode consistera aussi à confronter les champs théoriques et les données 
empiriques et à isoler les logiques propres des différents discours pour tenter de construire un caléidoscope des compréhensions de l'expérience et de l'angle de vue.

En cherchant une possibilité théorique cohérente avec l'expérience vécue telle que je l'entendais et la ressentais dans mes relations avec les personnes concernées, j'ai d'une part rencontré la phénoménologie (Depraz et al., 2011; Merleau-Ponty, 1945) et, d'autre part, les théories du sujet politique et de la subjectivité nomade (Braidotti, 2002, 2013). J'essaie grâce à elles de saisir l'évolution des subjectivités dans les modifications corporelles, et ce au travers des enjeux politiques et relationnels en présence. Les corps trans sont les lieux de multiples modifications corporelles: hormonothérapies, chirurgies, mais aussi transformations transgressives des esthétiques du genre, création de nouveaux modes d'expression ainsi que des reterritorialisations des corps vécus, psychologiques, symboliques, imaginaires. Le fait central est que les personnes trans ne remettent pas en question uniquement le genre, elles deviennent autres que le fameux destin biologique, elles sont créations humaines, œuvres biotechnologiques par l'utilisation des hormones. Elles mettent en crise le «naturel». Les travaux féministes anglo-saxons ont déjà mis en évidence combien l'expérience trans soulevait la question de l'humain dans une idée de posthumanité ${ }^{4}$ et de biotechnologies (Halberstam et al., 1995; Nurka, 2015).

Questionner le genre aujourd'hui ce n'est plus parler du féminin ou du masculin comme s'il s'agissait d'entités théoriques ou symboliques existant par elles-mêmes, ni seulement discuter la pertinence des stéréotypes sociaux et des représentations esthétiques. Questionner le genre c'est passer après Money, Stoller, De Beauvoir, Butler, Fausto-Sterling, Halberstam et tant d'autres. C'est mettre en perspective la binarité du système de genre comme élément constituant du genre, soit les relations de pouvoir et les hiérarchies sociales. C'est aussi prendre en compte ce qui se passe dans le monde réel, c'est-à-dire le fait que les demandes et les parcours des personnes trans ne se résument plus depuis longtemps à la binarité (Bockting, 2008; Herdt, 1994; Richards et al., 2016; Rimes et al., 2017), et c'est comprendre enfin que de parler de «transsexualité» en 2019 c'est manquer son objet.

Au paradigme de la très critiquée transsexual narrative qui imposait un récit typique d'une erreur sur le corps à corriger, d'une transformation et d'une mise en conformité anatomique et identitaire avec un genre unique, les corps trans opposent la multiplicité. Les corps et subjectivités trans d'aujourd'hui sont ceux de femmes trans, hommes trans, transgenres, non 
binaires, agenrés, bigenrés $^{5}$. Comme le montrent les travaux sociologiques et de santé publique récents, les identités, nominations et vécus du genre sont en mutation. La diversité des genres des personnes trans a comme dénominateur commun de ne pas être cisgenre, soit de ne pas se sentir convoquées par les injonctions sociales à correspondre à un type de genre défini pour soi par les autres en fonction du visible anatomique génital.

Pour envisager une clinique avec les personnes trans, la notion d'identité, à travers celle d'identité de genre, est à préciser afin de sortir d'une vision d'une identité immuable, stable et unique, à la faveur d'une subjectivité nomade et en devenir. J'insiste sur la nécessité conceptuelle de faire la différence entre identité et subjectivité. Si les deux concepts parlent tous deux de l'individualité d'une conscience réflexive incarnée et donc du Sujet, l'identité réfère à un processus d'affiliation, appartenance et reconnaissance d'un lien avec une communauté ou un groupe social. La subjectivité renvoie pour sa part au processus de définition de soi dans l'interface entre le corps et les autres/le social, alors que l'identité est le résultat de ce processus, une nomination de soi. Si l'on veut commencer à saisir quelque chose du genre il me semble utile d'envisager que le genre comme identité n'équivaut pas à la subjectivité de genre. Le premier est une négociation avec les normes qui finit par aboutir à une position dans une forme de stabilité; le second peut être variable selon les contextes, il est le ressenti et représenté de soi moment par moment. C'est dans les interstices entre identité et subjectivité que le devenir trans prend forme. Un devenir relationnel. La subjectivité n'est ellemême pas exempte des enjeux politiques et relationnels, comme cela a été relevé par de nombreuses auteures dans les champs des sciences humaines (Luhrman, 2006). Le Sujet comme posture d'énonciation, identité, est la part narrative de reconstruction de soi qui donnera sens au devenir et donc à l'histoire individuelle. Le Sujet parle "en contexte», il s'exprime dans des relations, à des autres qui ne sont pas une option mais une nécessité existentielle. Car, nous le savons tous, le Sujet (avec un grand «S») n'existe pas en soi, il n'est jamais qu'une fiction inspirée d'évènements réels et de sensations ressenties, à la faveur de ce qui est à sa disposition pour s'écrire et se réécrire (Ricœur, 1985), pour soi et pour et par les autres. Le Sujet accepte, choisit, change son identité, il se laisse guider par sa subjectivité toujours incarnée qu'il organise, écoute ou dénie. 


\section{Ressentir et devenir soi}

Les personnes trans ne croient pas qu'elles sont des «femmes dans un corps d'homme» ou des «hommes dans un corps de femme» - ceci est un acte de discours (pour se faire comprendre). Parfois un acte de discours sur lequel il y a collusion entre le monde médical et certaines personnes trans. Leurs récits de vie témoignent d'un douloureux processus pour pouvoir se penser en dehors du système de genre, car celui-ci structure le langage et la société et propose une binarité dont il n'est pas aisé de s'affranchir.

Le genre assigné à la naissance est vécu comme une négation et un désinvestissement de la corporéité. C'est un "genre de» corps qui n'est pas à soi; ce corps dans sa matérialité est toutefois reconnu comme le corps, et c'est ce qu'il exprime du genre (de la performance du genre, y compris au sens émotionnel, symbolique, esthétique) qui n'est pas reconnu et dès lors engendre souffrance, sentiment de profonde inadéquation et incohérence.

Une question fondamentale pour la clinicienne est celle de l'intentionnalité et du moteur du devenir. Je ne parle pas ici d'une autorité qui imposerait, sanctionnerait ou autoriserait une intervention médicale. Je veux parler du point de vue du Sujet, du point de départ du mouvement. Et je parle intentionnellement d'intentionnalité et non d' "origine» ou d' «étiologie», car justement, du point de vue du Sujet, c'est toujours la mise en scène créative d'une histoire de soi signifiante et organisée, et c'est ce qui est accessible dans notre cabinet et au travers notre écoute.

Or la narration de soi la plus fréquente se résume dans cette phrase prototypique: «ÇA A TOUjours ÉTÉ LÀ, JE ME SUIS TOUJOURS SENTI.E AINSI». C'est justement sur cette phrase que ma compréhension - à un moment donné - a changé de perspective. Si, au départ mon biais psychologique pouvait me faire entendre le mot "TOuJOuRs», c'est aujourd'hui le mot «SENTI. E» qui m'apparaît le plus fondamental. Si l'on écoute bien ce mot et que l'on en prend la mesure, il nous apparaît que l'individu contemporain, ici la personne trans, attribue une forme de statut de vérité de soi à ce qu'elle ressent corporellement et affectivement. Que ce ressenti constitue à la fois une direction et une force, qu'il a l'intention et le pouvoir de changer ce qui aurait été le destin dans une société où le genre ne serait que dichotomique et synonyme de morphologie.

La subjectivité trans est incarnée et cet aspect est central. L'affirmation d'une identité est certes fondamentale, mais elle est seconde, car elle est forcément précédée par une reconnaissance à l'intérieur de soi d'une sensation ou d'une émotion qui n'arrive pas à être associée harmonieusement à ce 
qui serait censé être. Si nous voulons penser en termes de genre, et non de sexe, nous devons le comprendre pour ce qu'il est: un système symbolique de pouvoir et de connotations constitué dans un processus circulaire entre performance (Butler, 1990) et traduction (Medico, 2016). Le genre est attribué à ces ressentis et connoté dans des tensions et hiérarchies. Les personnes trans témoignent des efforts continuels pour tenter de correspondre, pour canaliser les mouvements du corps, les postures, qui ne seraient pas compatibles avec le genre assigné. Ainsi lors d'une transition de genre, les hommes trans ne deviennent pas des hommes, ils embrassent le masculin et cessent de jouer à être une femme. Les femmes trans ne deviennent pas des femmes, elles cessent de jouer à être un homme et laissent leur féminité s'exprimer. Les personnes non binaires cessent de se conformer à un genre qui ne les représente pas et cessent de s'obliger à choisir entre l'un ou l'autre car les deux options, féminin/masculin, ne correspondent pas à ce qui est ressenti de soi émotionnellement, corporellement, socialement, esthétiquement. La transition relève de la nécessité, transformée en volonté, d'apaiser l'inconfort et d'être authentique avec soi et les autres.

Ce que nous pouvons comprendre est que l'aspect corporo-affectif n'est pas seulement un épiphénomène du devenir mais son origine, son moteur et son lieu. Ce corps ressentant est autopoïétique. D’ailleurs pour beaucoup de personnes que j'ai rencontrées, la sexualité et le plaisir sensuel et sexuel ne sont pas un désert impraticable, au contraire, ils représentent un lieu d'expérimentation de soi, dans son corps et/ou avec les autres. La sexualité est le lieu du comme si, un espace de jeu et d'expérimentation. Il faut peutêtre cesser de mettre sur le sexuel la gravité qu'y mettait le XIX ${ }^{e}$ siècle: ce n'est plus d'actualité, du moins pas pour tout le monde. La sexualité est un espace d'intime et de jeu permettant de coloniser des corps, de les ressentir, de les «genrer» et " dégenrer ${ }^{6} »$, d'habiter le territoire-corps d'une manière ou d'une autre, d'espérer des liens, de les vivre, d'être vu et d'exister pour l'autre. La subjectivité sexuelle au sens de la position de genre dans laquelle la personne se sent lorsqu'elle est sexuellement excitée exprime beaucoup du genre et de son devenir.

Le concept de devenir, par sa mouvance, son potentiel d'évocation des intensités et des corporéités s'est imposé. Comme le disent Deleuze et Guattari dans une phrase qui pourrait résumer tout ce que j'ai appris des personnes trans: 
Le devenir ne produit pas autre chose que lui-même. C'est une fausse alternative qui nous fait dire: ou bien on imite, ou bien on est. Ce qui est réel, c'est le devenir lui-même, le bloc de devenir, et non pas des termes supposés fixes dans lesquels passerait celui qui devient. (Deleuze et Guattari, 1980, p. 291)

Ce devenir peut, dans ses rapports au système de genre, prendre au moins quatre voies. Premièrement, il peut viser la construction d'un genre unique en adéquation avec les normes sociales à disposition (masculin, féminin) tel qu'on le retrouve chez les hommes trans, les femmes trans et la plupart des personnes cisgenres. Deuxièmement, il peut s'exprimer dans une alternance temporelle entre deux ou plusieurs formes de genre selon les contextes et les moments de vie, sans que cela soit à comprendre comme une indécision mais plus comme une multiplication des subjectivités de genre. Troisièmement, il s'exprime de plus en plus comme une intégration, sorte de métissage et, peut-être, dépassement du système de genre dans une position transgenre et/ou non binaire. Et, quatrièmement, il peut consister en une neutralité qui rejette le concept de genre, le masculin comme le féminin. Pour les personnes agenres, le système de genre en lui-même ne résonne pas et ne fait pas sens. Il ne s'agit donc pas de penser les personnes trans en termes de contenus, de références à un masculin ou un féminin, mais en termes de processus et devenirs.

\section{Concevoir et révéler}

Si l'ancrage du devenir est de l'ordre de la sensation-reconnaissance, il ne peut advenir sans les mots et les concepts: trouver des mots pour exprimer le vécu, pour se comprendre soi-même, pour avoir des figures de comparaison, pour savoir enfin que l'on n'est pas seul, pour savoir qui on est. Cette étape est décrite comme un deuxième moment clé de l'histoire personnelle, celui de la révélation. Par le fait même de lire ce que d'autres ont écrit, de chercher des informations sur la question trans, de pouvoir dire tout simplement le mot «transgenre» ou «non binaire», une partie importante de la souffrance s'apaise. L'insaisissable de la différence devient compréhensible, devient subjectivité. Internet est le lieu habituel de cette révélation qui permet d'entrevoir une possibilité d'existence pour soi et les autres. Un reportage télévisé, un blog ou une rencontre ont un effet de soulagement. À partir de ce moment-là, la lutte contre soi se modifie, elle se structure, les enjeux se 
dessinent. C'est toujours difficile, mais les incohérences se relativisent parce qu'elles prennent sens.

Il y a là un effet de cohorte et de contexte important. Pour cette raison il me semble hasardeux de définir la question trans en fonction du moment d'apparition de la demande de changement de genre ou de l'âge des personnes. Le contexte de vie est prépondérant et il a énormément changé depuis quelques années. Les jeunes non binaires nous renvoient aujourd'hui cette idée que les personnes trans plus âgées, nées et ayant vécu dans un autre monde, ne pouvaient que difficilement penser. Avant qu'une pensée hors binarité ne soit possible, l'expérience trans était vécue comme des tentatives désespérées de se situer dans un pôle ou l'autre de la binarité de genre, une prison dans laquelle les murs étaient trop étroits. Avec le concept de non binarité et de neutralité nait une possibilité de se situer quelque part et d'être reconnu dans sa complexité. Une vie sans devoir être constamment comparé à ce qu'on n'est pas, sans devoir être toujours défini par défaut: pas une femme biologique, pas un «vrai» homme. Une vie différente mais possible.

Vivre avec les autres nécessités de se faire comprendre, de traduire pour ceux qui n'entendent pas. Il faut résumer la complexité du genre et sa mouvance. La question du langage, surtout en français, qui est particulièrement binaire et qui donne un genre à toute chose, ne peut être déjouée que dans la métaphore. Au moment de la lutte contre soi, souvent en première partie des démarches thérapeutiques, la plus fréquente que le clinicien peut entendre est celle de la guerre et de la lutte. Les personnes trans, et surtout celles qui ne se reconnaissent pas dans un genre binaire, semblent se penser comme un lieu dans lequel une guerre se joue entre deux personnages, l'un masculin, l'autre féminin. Une guerre destructrice dans laquelle le sujet se demande s'il doit se tuer lui-même en tuant une partie de soi. Guerre dans laquelle il hésite parfois, renonce, revient, emprisonne, libère. Sortir de la guerre peut prendre plusieurs voies, celle d'une reconquête de territoire où un genre fait disparaître l'autre, où un genre unique s'impose, ou où s'instaure une cohabitation plus ou moins pacifique, non binaire. Le chemin dépendra de l'équilibre des genres ressentis mais aussi de la capacité de chacun à s'inscrire en dehors des schémas traditionnels du genre et des pressions à la normalisation. Le devenir trans sera donc variable et fonction des rapports au pouvoir et de l'espace de liberté possible pour chacun. Car les mécanismes de pouvoir régulateurs externes sont à l'œuvre et la stigmatisation et la violence fonctionnent bien. Ils attaquent les particules dissidentes, proposent 
des métaphores de maladies infectieuses et d'épidémie à juguler, mettent en acte violences, répressions, rejet.

\section{Affronter et changer}

Le devenir trans est aussi un parcours de violences vécues, de violences réelles. La plupart des personnes craignent d'être rejetées, violentées, abandonnées. Les images généralement véhiculées dans la société leur font peur. Cette peur est justifiée. Rappelons que les études font consensus sur ce point: les personnes trans sont plus à risque de vivre de la violence sous toutes ses formes, y compris le meurtre, et d'avoir un accès plus difficile à l'emploi, des conditions socio-économiques plus précaires (Reisner et al., 2016; Stewart et al., 2017).

Cette violence des autres sur soi est intériorisée en une souffrance avec soi - souffrance psychique, transphobie intériorisée, haine de soi. Parfois aussi elle se transforme en jouissance ${ }^{7}$. Fréquemment rapportée, cette souffrance prend différentes formes: la honte, la culpabilité, la dépression, le sentiment de ne pas exister, le manque de confiance en soi et dans ses ressentis, la peur des autres, le manque de confiance dans les autres voire, les sentiments de persécution, l'anxiété, la dépression, le suicide et les automutilations (White et al., 2015). Mais aussi le mouvement sadique contre une part de soi qui est jouée dans un des personnages genrés du scénario intérieur. Il est de plus en plus clair aujourd'hui que c'est l'introjection de la violence sociale et du déni d'existence qui entraîne la haine de soi et par conséquent les souffrances psychiques qui sont si fréquentes aujourd'hui chez les personnes trans.

Pour celleux ${ }^{8}$ qui ont dépassé ce sentiment d'être pris au piège et entrepris une transition, des difficultés avec le monde médical et psychiatrique sont rapportées. Le principal problème tient aux situations de monopole et d'abus de pouvoir qui ont été vécues et qui sont largement documentées aujourd'hui (Namaste, 2000; Sironi, 2003). Puis il y a les arrangements du quotidien, des relations de couple, familiales, professionnelles, amicales: comment vivre en secret, oser le révéler et se révéler au risque d'être rejeté? "Qui voudrait d'un travesti?», déclare une personne bigenrée, en dehors d'une rencontre sexuelle d'un soir? Les personnes trans souhaitent être reconnues et aimées, mais pas comme des monstres, pas comme des curiosités, pas comme des objets sexuels exotiques.

Une mélancolie sans autorisation culturelle de deuil, dirait Butler (2009), serait le propre des expériences minoritaires. Un minoritaire qui ne 
serait pas que numéraire mais qui serait de l'ordre de l'absence de modèles, de destins tout tracés, de possibilités de se penser avec les outils symboliques d'une société qui les nie ou les désubjectivise (Lenning et Buist, 2013). S’en libérer nécessite une force d'auto-affirmation particulière, voire exceptionnelle (Braidotti, 2002). Il s'agit de transformer l'abject et de se construire au-delà (Halperin, 2010).

Le genre, avec le système de genre, s'avère être un concept écran qui sert à communiquer une narration de soi. Cette narration de soi est dès le départ inadaptée à ce qu'elle essaie de dire. Elle est de l'ordre de la reconnaissance en soi et par les autres, d'un ressenti en dehors de ce qui était prévu par le destin social et biologique. Il ne s'agit pas que de parler du masculin et du féminin. Ces instances symboliques sont des manières de s'exprimer pour être compris. Il s'agit plus profondément d'une question de multitude, de liens, d'affects, de sentiments, de sensations qui sont réinterprétés selon une grammaire binaire, mais qui pourraient aussi prendre d'autres formes et qui les prennent d'ailleurs de plus en plus.

\section{Les corps trans modifient-ils nos subjectivités?}

Les corps trans transforment plus que les genres. Ils ne sont pas que la réplique d'une idée du féminin et du masculin. Ces corps et ces subjectivités donnent à réfléchir sur le devenir versus le destin et sur la relative fixité du monde dans ce qu'il y aurait de plus «naturel»: le corps.

Le corps ne change pas que dans son apparence, il se modifie dans son ressenti et ces modifications du ressenti sont autant la conséquence que la cause du devenir, ils sont le devenir. Il n'y a pas ici qu'une affaire d'apparence à modifier ou de genre à inverser ou transformer, c'est une modification profonde des perceptions du monde et de la manière de le ressentir. Une construction nouvelle se développe dans ces expériences. Les modifications corporelles sont tout autant volontaires - comme l'hormonothérapie, la chirurgie - qu'involontaires - comme les modifications du schéma corporel et du ressenti qui parfois précèdent et accompagnent les modifications volontaires. Il n'est pas tout à fait juste de dire que les personnes trans modifient leur corps: les modifications corporelles (volontaires et involontaires) les modifient, tout comme le vieillissement ne modifie pas nos corps et nos subjectivités, nos corps nous vieillissent. Le corps n'est pas une matière informée mais informante et transformante.

L'expérience trans défie le dualisme corps/esprit et montre la limite de certaines de nos postures psychologiques profondément ancrées dans ce 
dualisme. Elle questionne les fondements ontologiques de nos théories. Elle nous parle des matérialités signifiantes qui se sentent à l'étroit entre les deux grands paradigmes essentialistes et constructivistes. Il semble fou de continuer à penser cela comme une exception. Toutes ces expériences trans sont visibles et transformatives. Elles sont dans l'espace social et modifient les imaginaires, les esthétiques, les érotiques ${ }^{9}$ et peut-être aussi les corps des subjectivités contemporaines. Une posture théorique, le nouveau matérialisme ${ }^{10}$, se développe actuellement dans le champ des théories critiques, de l'art et de la philosophie. Elle tente de repenser la subjectivité avec la matière. En gardant les apports du socio-constructivisme, notamment autour des analyses des conditions de production des savoirs, elle propose d'éviter le relativisme trop absolu qui oublierait des aspects fondamentaux du Sujet: l'incarnation, le potentiel autopoïétique et de transformation du monde avec les processus non humains dans lesquels il baigne.

Denise Medico

medico.denise@uqam.ca

\section{Notes}

1. Si l'estimé le plus fiable de la proportion des personnes trans semble situer celle-ci autour de 0,39\% (Meerwijk et Sevelius, 2017), les travaux épidémiologiques chez les 13-18 ans relèvent une proportion bien plus élevée, soit entre 0,7 et 2,7 \% (Clark et al., 2014; Herman et al., 2017; Rider et al., 2018).

2. Sa rhétorique est d'ailleurs très proche de celle de la sociobiologie de la sexualité, tel qu'on peut la lire dans l'ouvrage de Donald Symons (1996), Du sexe à la séduction: L'évolution de la sexualité humaine.

3. Pronom référant aux personnes neutres et non binaires.

4. Le posthumanisme engage une réflexion sur l'impact des biotechnologies dans la modification de la notion d'humain, mais aussi sur la généralisation que la notion d'humain/ humanisme a promue autour du masculin européen comme inquestionné des représentations de l'humain. Voir Braidotti, 2013.

5. Le concept de «two-spirit» a été proposé pour parler de certaines configurations du genre multiple dans certains groupes autochtones.

6. Nous reprenons ici une formulation usuelle dans les communautés trans et les études critiques.

7. Je songe ici au propos de Julia Kristeva sur l'abjection et l'«alchimie qui transforme la pulsion de mort en sursaut de vie, de nouvelle signifiance» (1980, p. 22).

8. Néologisme soulignant la diversité des genres, référant tant aux personnes non binaires qu'aux hommes et aux femmes.

9. Les fantasmes sur des personnes trans se sont imposés ces dernières années dans la population masculine cisgenre, la pornographie et le travail du sexe en étant des indicateurs. Voir à cet effet Escoffier, 2011.

10. "From this perspective we drew attention to a new materialist predilection for a more phenomenological approach to embodiment. In addition to focusing on the way power 
constitutes and is reproduced by bodies, phenomenological studies emphasize the active, self-transformative, practical aspects of corporeality as it participates in relationships of power. They find bodies exhibiting agentic capacities in the way they structure or stylize their perceptual milieu, where they discover, organize, and respond to patterns that are corporeally significant» (Coole et al., 2010, p. 19-20).

\section{Références}

Bailey, J. M. (2003). The Man Who Would Be Queen. The Science of Gender-bending and Transsexualism. Washington: Joseph Henry Press.

Blanchard, R. (1989). The concept of autogynephilia and the typology of male gender dysphoria. Journal of Nervous and Mental Disease, 177 (10), 616-623.

Bockting, W. O. (2008). Psychothérapie et expérience de vie réelle: de la dichotomie à la diversité de genre. Sexologies, 17, 211-224.

Bolin, A. (1994). Transcending and transgendering: Male-to-female transsexuals, dichotomy and diversity. Dans G. Herdt (dir.), Third Sex, Third Gender: Beyond Sexual Dimorphism in Culture and History (p. 447-486). New York: Zone Books.

Braidotti, R. (2002). Metamorphoses: Towards a Materialist Theory of Becoming. Malden : Polity Press/Blackwell Publishing.

Braidotti, R. (2013). The Posthuman. Cambridge: Polity Press.

Butler, J. (1990). Gender Trouble: Feminism and the Subversion of Identity. New York/London: Routledge.

Butler, J. (2009). Le transgenre et «les attitudes de révolte». Dans M. David-Ménard (dir.), Sexualités, genres et mélancolie. S'entretenir avec Judith Butler (p. 13-33). Paris: Campagne Première.

Clark, T. C., Lucassen, M. F. G., Bullen, P., et al. (2014). The health and well-being of transgender high school students: results from the New Zealand Adolescent Health Survey (Youth'12). Journal of Adolescent Health, 55 (1) : 93-99.

Coole, D. et Frost, S. (2010). Introducing the New Materialisms. Dans D. Coole et S. Frost (dir.), New Materialisms. Ontology, Agency, and Politics (p. 1-43). Durham and London: Duke University Press.

Depraz, N., Varela, F. J. et Vermersch, P. (2011). À l'épreuve de l'expérience. Pour une pratique phénoménologique. Bucarest: Zetabooks.

Deleuze, G. et Guattari. F. (1980). Capitalisme et schizophrénie. 2. Mille Plateaux. Paris: Éditions de Minuit.

Escoffier, J. (2011). Imagining the She/Male: Pornography and the Transsexualization of the Heterosexual Male. Studies in Gender and Sexuality, 12 (4), 268-281.

Halberstam, J. et Livingston, I. (1995). Introduction: Posthuman Bodies. Dans J. Halberstam et I. Livingston (dir.), Posthuman Bodies (p. 1-20). Bloomington : Indiana University Press.

Halperin, D. (2010). Que veulent les gays? Essai sur le sexe, le risque et la subjectivité. Paris: Éditions Amsterdam.

Hérault, L. (2010). Usages de la sexualité dans la clinique du transsexualisme. L'autre, cliniques, cultures et sociétés, 11 (3), 278-290.

Herdt, G. H. (1994). Introduction: Third sexes and third genders. Dans G. H. Herdt (dir.), Third Sex, Third Gender: Beyond Sexual Dimorphism in Culture and History (p. 21-81). New York : Zone Book.

Herman, J., Flores, A. R., Brown, T. N. T., Wilson, B. D. M et Conron, K. (2017). Age of individuals who identify as transgender in the United States. Repéré à https://williamsinstitute.law.ucla.edu/wp-content/uploads/TransAgeReport.pdf. 
Hirschauer, S. (1997). The medicalization of gender migration. International Journal of Transgenderism, 1 (1). Repéré à http://www.symposion.com/ijt/ijtc0104.htm.

Kristeva, J. (1980). Pouvoirs de l'horreur. Paris: Seuil.

Lenning, E. et Buist, C. L. (2013). Social, psychological and economic challenges faced by transgender individuals and their significant others: gaining insight through personal narratives. Culture, Health \& Sexuality, 15 (1), 44-57.

Luhrmann, T. M. (2006). Subjectivity. Anthropological Theory, 6 (3), 345-361.

Medico, D. (2014). Éléments pour une psychothérapie adaptée à la diversité trans*. Cahiers critiques de thérapie familiale et pratiques de réseau, 52, 109-137.

Medico, D. (2016). Repenser le genre. Une clinique avec les personnes trans. Chêne Bourg: Georg/Médecine et hygiène.

Medico, D. et Volkmar, E. (2016) La Fondation Agnodice: pour une société plus juste à l'égard des personnes transgenres. Nouvelles Questions féministes, 35 (1), 182-186.

Medico, D., Chapot, F., Volkmar, E., Santiago Delefosse, M. (2009). Adapter la prévention IST/VIH-sida dans des contextes de liminarité sociale, éthique et enjeux. Enquête auprès d'un groupe de personnes transgenres travailleuses du sexe brésiliennes en Suisse Romande. Dans L. Chamberlain, B. Frank et J. Ristock (dir.), Minorités sexuelles et constructions du genre (p. 335-358). Montréal: Presses de l'Université du Québec.

Meerwijk, E. et Sevelius, J. M. (2017) Transgender Population Size in the United States: a Meta-Regression of Population-Based Probability Samples. American Journal of Public Health, 107(2), 1-8.

Merleau-Ponty, M. (1945). Phénoménologie de la perception. Paris: Gallimard.

Namaste, V. (2000). Invisible Lives. The Erasure of transsexuals and transgendered people. Chicago/London: University of Chicago Press.

Nurka, C. (2015). Animal Techne. Transing Posthumanism. TSQ: Transgender Studies Quarterly, 2, 209-226.

Richards, C., Nouman, W. P., Seal, L., Barker, J., Nieder, T. O. et T'Joen, G. (2016). Non binary or genderqueer genders. International Review of Psychiatry, 28 (1), 95-102.

Rider, G. N., McMorris, B. J., Gower, A. L. et al. (2018). Health and Care Utilization of Transgender and Gender Nonconforming Youth: A Population-Based Study. Pediatrics, 141 (3): e20171683.

Reisner, S. L., Poteat, T., Keatley, J., Cabra, M., Mothopeng, T., Dunham, E., Holland, C. E., Max, R. et Baral, S. D. (2016). Global health burden and needs of transgender populations; a review. Lancet, 388 (100042), 412-436.

Stewart, L., O'Halloran, P. et Oates, J. (2017). Investigating the social integration and wellbeing of transgender individuals: A meta-synthesis. International Journal of Transgenderism, 18 (3), 1-13.

Reisner, S. L., Poteat, T., Keatley, J., Cabra, M., Mothopeng, T., Dunham, E., Holland, C. E., Max, R. et Baral, S. D. (2016). Global health burden and needs of transgender populations; a review. Lancet, 388 (100042), 412-436.

Ricœur, P. (1985). Temps et récit III: Le temps raconté. Paris: Seuil.

Rimes, K. A., Goodship, N., Ussher, G., Baker, D. et West, E. (2017). Non-binary and binary transgender youth: Comparison of mental health, self-harm, suicidality, substance use and victimization experiences. International Journal of Transgenderism, 18 (3), 1-13.

Sironi, F. (2003). Maltraitance théorique et enjeux contemporains de la psychologie clinique: les nouveaux défis éthiques. Pratiques psychologiques, 4, 3-13.

White H., J. M., Reisner, S. L. et Pachankis, J. E. (2015). Transgender stigma and health: A critical review of stigma determinants, mechanisms, and interventions. Social Science and Medicine, 147, 222-231. 